\title{
First-time sports-related concussion recovery revisited: management changes and impact on recovery
}

https://doi.org/10.1515/jom-2020-0106

Received April 30, 2020; accepted August 10, 2020;

published online January 29, 2021

\begin{abstract}
Context: Concussion is among the most common injuries in athletes. Over the past 10 years, concussion knowledge has expanded, and guidelines for management have changed. Recent changes include recommendations against strict mental and physical rest after sustaining a concussion, a better recognition of preexisting conditions predisposing protracted concussion recovery, and the addition of preliminary patient counseling and education about the expected course of concussion recovery.

Objective: To assess the impact of changes in concussion management on the duration of symptoms in middle school and high school athletes after sustaining a firsttime, sports-related concussion.

Methods: A retrospective review was performed of medical records of athletes who sustained a concussion between 2016 and 2018 and were treated by the same primary care physician (J.M.N) using the revised approach to concussion management described. Patients were included if they were 11 to 18 years old and had a diagnosis of first-time concussion sustained while playing organized sports. Athletes who sustained a concomitant injury or sustained a concussion outside of organized sports were excluded from the study;
\end{abstract}

*Corresponding author: John M. Neidecker, DO, ATC, Orthopaedic Speciaists of North Carolina, 11200 Governor Manly Way, Suite 309, Raleigh, NC 27614-8599, USA; and School of Osteopathic Medicine, Campbell University, Buies Creek, NC, USA,

E-mail: neidy13@yahoo.com

David B. Gealt, DO, Cooper Bone and Joint Institute, Cooper University Hospital, Camden, NJ, USA

Kathryn Lambert, DO, School of Osteopathic Medicine, Rowan University, Stratford, NJ, USA

John R. Luksch, DO, Rothman Institute, Philadelphia, PA, USA Martin D. Weaver, MD, Miller School of Medicine, University of Miami, Miami, FL, USA athletes lost to follow-up or with incomplete forms were also excluded. Each athlete's length of symptomatic time from his or her concussion was calculated. This data was then compared with a previously-published data set of athletes who sustained a concussion between 2011 and 2013, with the same inclusion criteria but a different approach to concussion management.

Results: A total of 110 male and 72 female athletes $(\mathrm{N}=182)$ met the study's eligibility criteria. Collectively, athletes of both sexes from the 2016-2018 data set reported a higher incidence of preexisting conditions associated with prolonged concussion recovery compared with the data set of 2011-2013 athletes. However, both sexes from the 2016-2018 data set also reported a shorter median duration of concussion symptoms compared with the 2011-2013 athletes (median duration 5 vs. 11 days for male athletes and 7 vs. 28 days for female athletes, respectively; $\mathrm{p}<0.001$ ).

Conclusion: Recent changes in concussion management have led to decreased duration of symptoms among 11- to 18-year-old athletes with first-time, sports-related concussions. These management changes include advocating for early activity, recognizing preexisting conditions, and educating athletes about the realities of concussion recovery.

Keywords: concussion management; high school athletes; preexisting conditions; student athletes.

Since 2013, there have been numerous publications and guidelines advancing concussion knowledge and treatment. ${ }^{1-18}$ More recent consensus statements have advocated for the abolishment of prolonged total rest or 'cocoon therapy' as a treatment for concussion. ${ }^{1,2}$ Current position statements on concussion also recognize that persistent postconcussive symptoms (PPCS) may be mistakenly attributed to concussion and are more likely linked to premorbid conditions. ${ }^{1}$ Therefore, it is critical to recognize and screen for preexisting conditions when evaluating and treating patients with concussion. ${ }^{2}$ Other publications suggest that patient perception of concussion and 
catastrophizing the injury may increase anxiety and affect recovery. ${ }^{3,4}$ Consequently, preliminary patient counseling, including education about the realities of concussion and the normal course of symptom resolution, may mitigate anxiety and improve concussion recovery.

We previously published a study ${ }^{5}$ demonstrating a longer recovery time from concussion symptoms in female athletes when compared with male athletes, ages 11-18 years old. That study $^{5}$ was a retrospective chart analysis of patients who sustained a concussion from 2011 to 2013. Included were athletes who sustained a first-time, sport-related concussion (SRC) with no other associated injuries. Because guidelines changed in SRC management after 2013, athletes seen during that prior period were treated differently than they are today. One of the major differences in SRC management is the recommendation for athletes to undergo "strict rest" before $2013^{19}$ and engage in "active rest" after new SRC guidelines were published in 2017. ${ }^{1}$ Strict rest instructs athletes to refrain from any physical or cognitive activity until concussion symptoms resolve, ${ }^{6}$ while active rest recommends that athletes initiate low intensity physical or cognitive activity at 24 to 48 hours following an SRC. ${ }^{1}$

The purpose of our current study was to determine whether these changes in concussion management have hastened the resolution of symptoms in first-time sports-related concussions among middle school and high school athletes.

\section{Methods}

\section{Design}

A retrospective medical records review was conducted on athletes who sustained a concussion between 2016 and 2018 and were seen at a single sports medicine practice in Raleigh, North Carolina. An outside healthcare provider (i.e., certified athletic trainer, primary care/emergency physician) referred nearly all athletes to the practice. Athletes were evaluated and treated by a single primary care physician (J.M.N.) with a Certificate of Added Qualification in sports medicine. This data was compared with data collected from the previous study ${ }^{5}$ published in 2017, which analyzed concussions sustained between 2011 and 2013 and in which J.M.N. was one of three treating physicians. The Campbell University Institutional Review Board deemed this study exempt.

\section{Criteria}

Athletes were included if they were between the ages of 11 and 18 years and had sustained a first-time concussion while playing organized sports. Athletes who sustained a concomitant injury with a concussion (e.g., nasal fracture, eye injury) or sustained a concussion outside of organized sports (e.g., gym class, backyard play, motor vehicle accident) were excluded from the study. Athletes lost to followup or with incomplete forms were also excluded.

\section{Collected data}

Demographic information (age, sex, and sport) and preinjury medical history of migraines/frequent headaches, learning disability, visual problems, and mental health problems were collected. Athletes were considered positive for a preinjury problem if they disclosed a previous relevant diagnosis or reported regular/frequent problems/issues before the concussion even if they were never given a formal diagnosis (e.g., migraine, vestibular/ocular dysfunction, anxiety, and depression). The length of time that each athlete was symptomatic was calculated using the documented medical record information from follow-up office visits. Concussion symptoms were screened and monitored using the Sport Concussion Assessment Tool-5th Edition (SCAT5) or Immediate Post-Concussion Assessment and Cognitive Testing (ImPACT). The criterion for symptom resolution was documentation that the athlete returned to baseline symptoms (before concussion). Baseline levels were determined through patient self-reporting.

\section{Data analysis}

All statistical tests were performed using Statistical Package for the Social Sciences (IBM). Independent t-tests were used to compare the mean age between the sexes, and the Mann-Whitney test was used to compare the ranks of asymptomatic days. Medians were evaluated rather than means for asymptomatic days because the data were not normally distributed, and thus, assessing the means would skew the results. $\chi^{2}$ tests were used to compare differences in the existence of preexisting conditions; $\mathrm{p}<0.05$ was considered statistically significant.

\section{Results}

Of the 364 medical records reviewed from 2016 to 2018, 182 athletes (110 male athletes and 72 female athletes) met inclusion criteria. History of previous concussion and concussion sustained outside of organized sports was the most common reason for excluding the study. 
Table 1: Gender comparisons of athletes in 2016-2018 ( $N=182)$.

\begin{tabular}{|c|c|c|c|}
\hline Characteristic & $\begin{array}{r}\text { Male athletes } \\
(n=110)\end{array}$ & $\begin{array}{r}\text { Female athletes } \\
\qquad(n=72)\end{array}$ & p-value ${ }^{a}$ \\
\hline Mean (SD) age, years & $15.3(1.45)$ & $15.2(1.41)$ & 0.904 \\
\hline \multicolumn{4}{|c|}{ Pre-injury medical history, $\%$} \\
\hline $\begin{array}{l}\text { Migraines/frequent } \\
\text { headaches }\end{array}$ & 26.4 & 38.9 & 0.053 \\
\hline Visual problems & 10.9 & 20.8 & 0.053 \\
\hline Learning disability & 17.3 & 8.3 & 0.065 \\
\hline $\begin{array}{l}\text { Mental problems } \\
\text { (anxiety/depression) }\end{array}$ & 6.4 & 19.4 & 0.007 \\
\hline $\begin{array}{l}\text { No. of days reporting } \\
\text { symptoms, median }\end{array}$ & 5 & 7 & 0.002 \\
\hline
\end{tabular}

${ }^{a}$ Statistically significant at $p<0.05$.

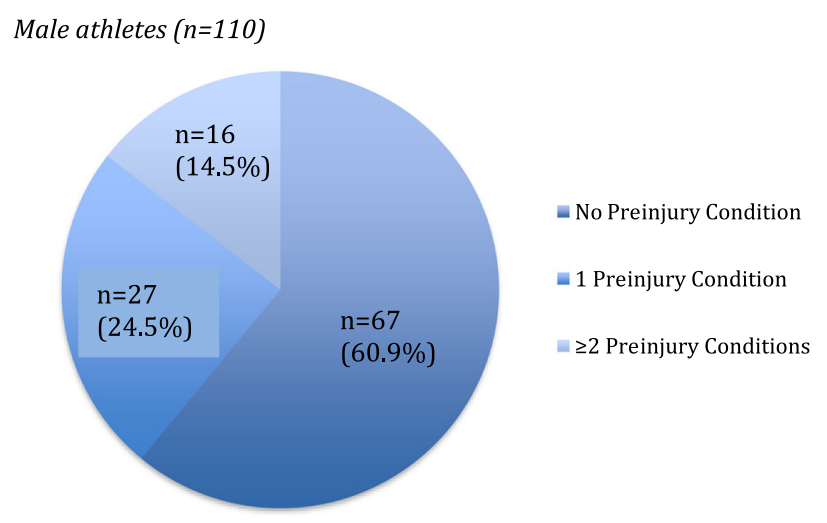

Female athletes $(n=72)$

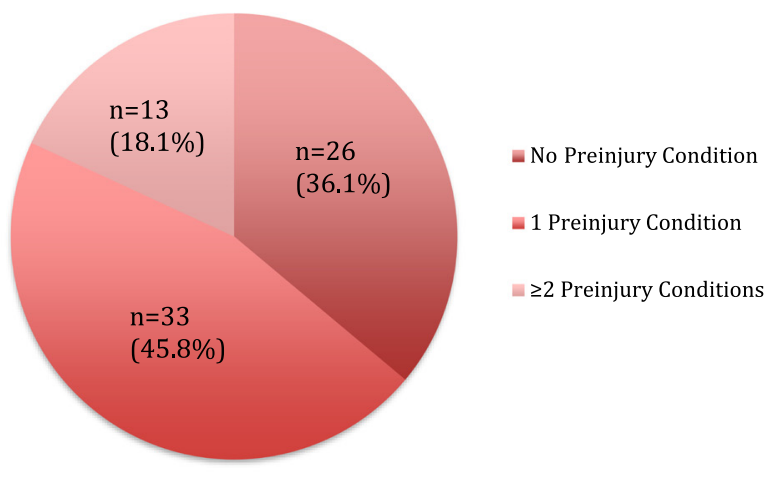

Figure 1: Percentage of male (blue, top; $n=110$ ) and female (red, bottom; $n=72$ ) athletes with pre-injury conditions in the 2016-2018 data set.

Table 1 shows the sex, age, and medical history distribution of athletes from the 2016-2018 data set. Within the sex groups, no significant difference was found in mean age (boys, 15.3 years vs. girls, 15.2 years; $\mathrm{p}=0.904$ ) nor in history of migraines/frequent headaches, visual problems, or learning disability between male and female athletes before injury (Table 1). Female athletes had a significantly greater incidence of preinjury history of mental health problems (anxiety/depression) than male athletes (19.4 vs. $6.4 \%$, respectively; $\mathrm{p}=0.007$ ). Figure 1 shows that, collectively, female athletes had a higher incidence of preinjury conditions than male athletes; 33 of 72 (45.8\%) female athletes had one preinjury condition, and 13 of 72 (18.1\%) female athletes had two or more preinjury conditions. These results contrasted with 27 of 110 (24.5\%) male athletes with one preinjury condition and 16 of 110 (14.5\%) male athletes who had two or more preinjury conditions, respectively. In broader terms, 46 of $72(63.8 \%)$ of female athletes compared with 43 of 110 (39.1\%) of male athletes had one or more preinjury conditions.

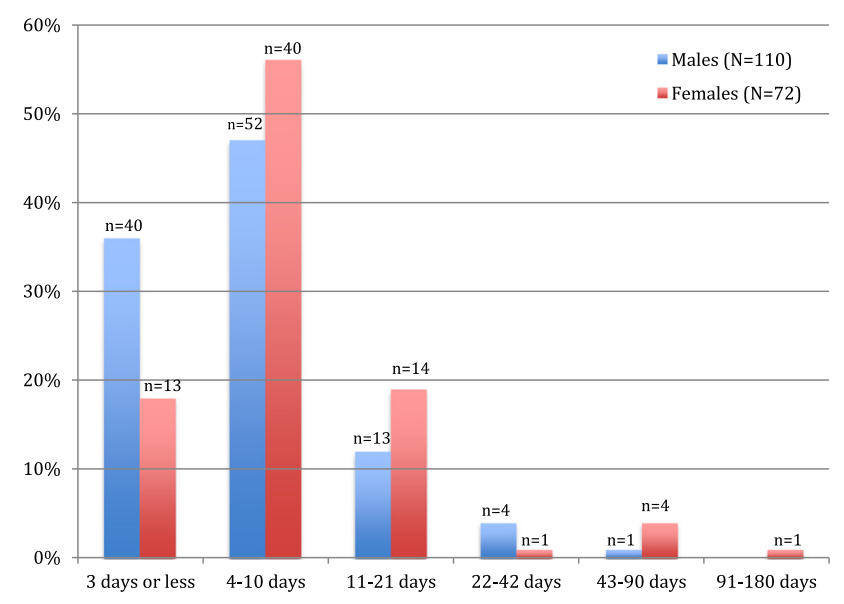

Figure 2: Comparison the percentage of male $(n=110)$ and female $(n=72)$ athletes in the $2016-2018$ study vs. duration of reported symptoms.

When analyzing the 2016-2018 data, a significant difference was found between male athletes and female athletes in the duration of symptoms (median, five days vs. seven days, respectively; $\mathrm{p}=0.007$ ). Our data revealed that 93 of 110 male athletes (84.5\%) experienced recovery of symptoms by 10 days compared with 53 of 72 female athletes (73.6\%, Figure 2). However, by three weeks, recovery normalized between sexes, as 105 of 110 male athletes (95.4\%) and 67 of 72 female athletes (93.1\%) experienced recovery of symptoms (Figure 2). In both male and female athletes, preinjury conditions were associated with a higher incidence of prolonged symptom duration beyond median recovery. This association was more exaggerated in the male subset. Male athletes displayed a $65.7 \%$ chance of symptom recovery by five days if there was no history of preinjury conditions compared with a $37.5 \%$ chance of symptom recovery by five days if there was a history of preinjury conditions (Table 2). 
Table 2: Symptom recovery based on sex and preexisting conditions among 2016-2018 male and female athletes $(\mathrm{N}=182)$.

\begin{tabular}{|c|c|c|c|}
\hline \multicolumn{4}{|l|}{ Male athletes, $n=110$} \\
\hline $\begin{array}{l}\text { No. of preexisting } \\
\text { conditions }\end{array}$ & $\mathbf{n}$ & $\begin{array}{r}\text { Chance of having } \\
\text { symptoms } \\
\leq 5 \text { days, } \%\end{array}$ & $\begin{array}{r}\text { Chance of having } \\
\text { symptoms } \\
>5 \text { days, } \%\end{array}$ \\
\hline None & 67 & 65.7 & 34.3 \\
\hline Learning disability only & 11 & 45.5 & 54.5 \\
\hline $\begin{array}{l}\text { Migraine/frequent } \\
\text { headaches only }\end{array}$ & 14 & 35.7 & 64.3 \\
\hline Vision problems only & 2 & 0 & 100 \\
\hline $\begin{array}{l}\text { Mental problems } \\
\text { (anxiety/depression) only }\end{array}$ & 0 & $\mathrm{~N} / \mathrm{A}$ & $\mathrm{N} / \mathrm{A}$ \\
\hline 1 total & 27 & 37 & 63.0 \\
\hline $2+$ total & 16 & 37.5 & 62.5 \\
\hline \multicolumn{4}{|l|}{ Female athletes, $n=72$} \\
\hline $\begin{array}{l}\text { No. of preexisting } \\
\text { conditions }\end{array}$ & $\mathbf{n}$ & $\begin{array}{r}\text { Chance to have } \\
\text { symptoms } \\
\leq 7 \text { days, } \%\end{array}$ & $\begin{array}{r}\text { Chance to have } \\
\text { symptoms } \\
>7 \text { days, } \%\end{array}$ \\
\hline None & 26 & 69.2 & 30.8 \\
\hline Learning disability only & 4 & 0 & 100 \\
\hline $\begin{array}{l}\text { Migraine/frequent } \\
\text { headaches only }\end{array}$ & 18 & 44.4 & 55.6 \\
\hline Vision problems only & 4 & 25 & 75 \\
\hline $\begin{array}{l}\text { Mental problems } \\
\text { (anxiety/depression) only }\end{array}$ & 7 & 71.4 & 28.6 \\
\hline 1 total & 33 & 54.5 & 45.5 \\
\hline $2+$ total & 13 & 46.2 & 53.8 \\
\hline
\end{tabular}

Table 3 compares middle school (11 to 13 years old) vs. high school (14 to 18 years old) athletes from 2016 to 2018, showing no statistically significant difference in length of reported symptoms between age groups. When comparing sex and the sport played, we also found no statistically significant differences in the resolution of concussion symptoms in the 2016-2018 data set (Figure 3).

Table 3: Age comparison among 2016-2018 male and female athletes $(\mathrm{N}=182)$.

\begin{tabular}{lrrr}
\hline Age, years & $\mathbf{n}$ & $\begin{array}{r}\text { No. of days reporting } \\
\text { symptoms, median }\end{array}$ & p-value $^{\mathrm{a}}$ \\
\hline Male athletes & 110 & & \\
$11-13$ & 9 & 5 & 0.208 \\
$14-18$ & 101 & 5 & \\
Female athletes & 72 & 6 & 0.739 \\
$11-13$ & 8 & 7 & \\
$14-18$ & 64 & & \\
\hline
\end{tabular}

${ }^{a}$ Statistically significant at $p<0.05$.

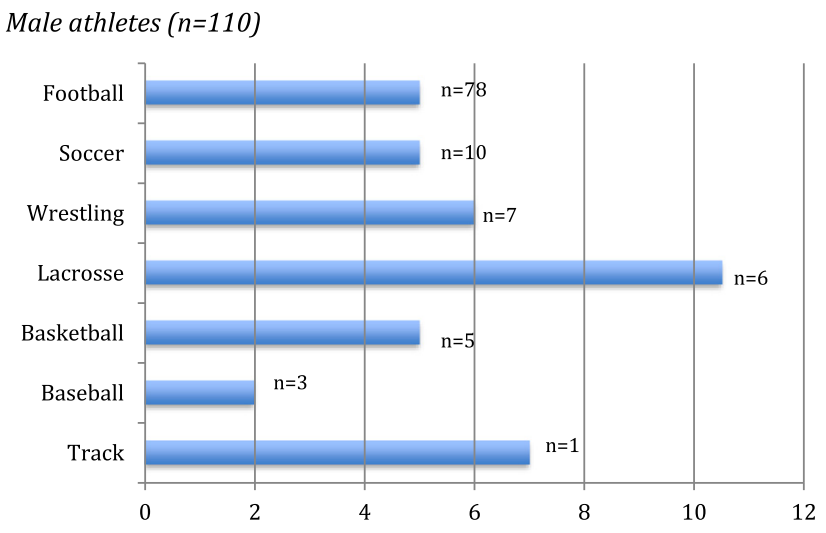

Female athletes $(n=72)$

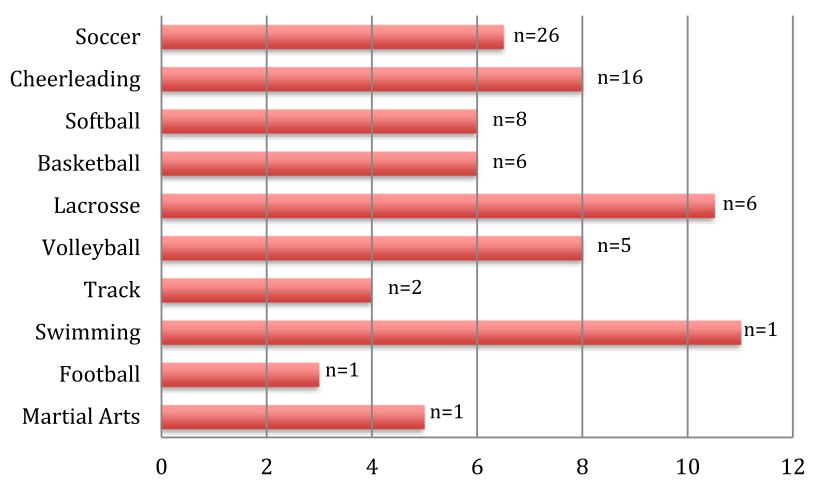

Figure 3: Sports participation in 2016-2018 vs. median days of reported symptoms in male (blue, top; $\mathrm{n}=110$ ) and female (red, bottom; $n=72$ ) athletes.

Data from athletes treated from $2011-2013^{5}$ were compared with the new data set of athletes from 2016-2018. Table 4 shows a comparison of sex, age, and medical history between the athletes in the two studies. Age between the two data sets showed a statistical difference; the mean age of male athletes was 14.8 years old in the 2011-2013 study ${ }^{5}$ and 15.3 years old in the 2016-2018 study ( $\mathrm{p}=0.049$ ). Likewise, the mean age of the female athletes was also significantly different between the two data sets; the mean age of the female athletes was 14.5 years old in the 2011-2013 study ${ }^{5}$ and 15.2 years old in the 2016-2018 study $(\mathrm{p}=0.006)$.

No statistically significant difference was found in male athletes in preinjury medical history of visual problems or learning disabilities between the $2011-2013^{5}$ and the 2016-2018 data sets. No statistically significant difference was found in female athletes in preinjury history of learning disability between the $2011-2013^{5}$ and the 2016-2018 data sets. A statistically significant difference was found in preinjury history of vision problems between the female athletes in the $2011-2013^{5}$ and the 2016-2018 data sets ( 4.6 vs. $20.8 \%$, respectively; $p=0.001$ ). 
Table 4: Comparison between 2011-2013 vs. 2016-2018 athletes. ${ }^{a}$

\begin{tabular}{|c|c|c|c|c|c|c|}
\hline \multirow[t]{2}{*}{ Characteristic } & \multicolumn{3}{|c|}{ Male athletes } & \multicolumn{3}{|c|}{ Female athletes } \\
\hline & $\begin{array}{r}2011-2013 \\
(n=110)\end{array}$ & $\begin{array}{r}2016-2018 \\
(n=110)\end{array}$ & p-value & $\begin{array}{r}2011-2013 \\
(n=102)\end{array}$ & $\begin{array}{r}2016-2018 \\
(n=72)\end{array}$ & p-value \\
\hline Mean (SD) age, years & $14.8(1.82)$ & $15.3(1.45)$ & 0.049 & $14.5(1.58)$ & $15.2(1.41)$ & 0.006 \\
\hline \multicolumn{7}{|l|}{ Pre-injury medical history, \% } \\
\hline Migraines/frequent headaches & 3.6 & 26.4 & 0.000 & 13.8 & 38.9 & 0.000 \\
\hline Visual problems & 4.5 & 10.9 & 0.064 & 4.6 & 20.8 & 0.001 \\
\hline Learning disability & 18.2 & 17.3 & 0.500 & 11.9 & 8.3 & 0.253 \\
\hline Mental problems (anxiety/depression) & 0.9 & 6.4 & 0.033 & 4.6 & 19.4 & 0.003 \\
\hline No. of days reporting symptoms, median & 11 & 5 & 0.000 & 28 & 7 & 0.000 \\
\hline
\end{tabular}

${ }^{a}$ Statistically significant at $p<0.05$.

Statistically significant differences were found in both preinjury histories of migraines/frequent headaches and mental health problems (anxiety/depression) in male athletes between the 2011-2013 $3^{5}$ and the 2016-2018 data sets $(\mathrm{p}=0.000$ and $\mathrm{p}=0.033$, respectively). This preinjury difference was also noted between the female athletes when the 2011-2013 data set ${ }^{5}$ with the 2016-2018 data set ( $\mathrm{p}=0.000$ and $\mathrm{p}=0.003$, respectively).

There was a significant difference in the duration of symptoms between the male athletes in the 2011-2013 study ${ }^{5}$ vs. the 2016-2018 data sets (median, 11 vs. 5 days, respectively; $p<0.001)$. Our data revealed that 82 of the 110 (74.5\%) male athletes in the 2011-2013 data set ${ }^{5}$ experienced recovery of concussion symptoms by three weeks compared with 105 of the 110 (95.4\%) male athletes in the 2016-2018 data set (Figure 4).

A greater significant difference was found between the female athletes in the $2011-2013^{5}$ and $2016-2018$ data sets in the duration of symptoms (median, 28 vs. 7 days, respectively; $\mathrm{p}<0.001)$. Our data revealed that 42 of the 102 (41.2\%) 2011-2013 ${ }^{5}$ female athletes experienced recovery of

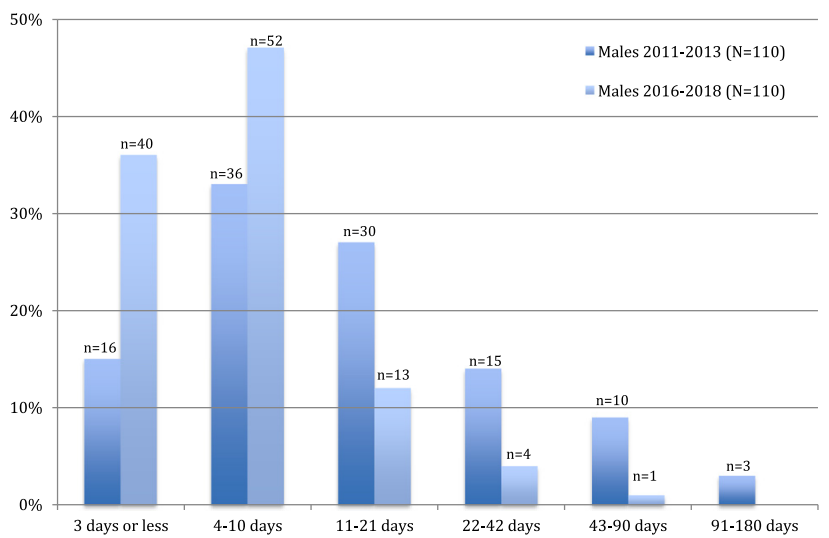

Figure 4: Percentage of male athletes in the 2011-2013 study ${ }^{5}$ compared with 2016-2018 study vs. duration of reported symptoms.

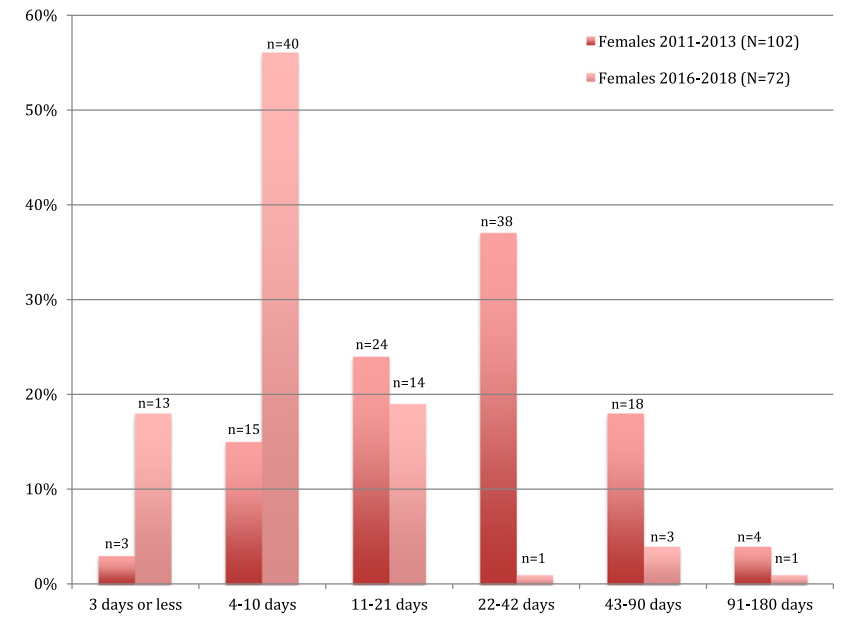

Figure 5: Percentage of female athletes in the 2011-2013 study ${ }^{5}$ compared with 2016-2018 study vs. duration of reported symptoms.

concussion symptoms by three weeks compared with 67 of the 72 (93.1\%) 2016-2018 female athletes (Figure 5).

\section{Discussion}

As evidence-based medicine has advanced our understanding of SRC, guidelines have changed, thus altering concussion management. The current study reflects how applied changes in the management of SRC can significantly improve concussion symptom recovery. The single primary care sports medicine physician (J.M.N.) providing the 2016-2018 data for this study recognizes that his management of SRC has changed significantly in three different ways since 2013: (1) recommendations against strict mental and physical rest after sustaining a concussion, (2) a better recognition of preexisting conditions that have been associated with protracted concussion recovery, (3) initial patient counseling and education about the realities of concussion and the normal course of symptom 
resolution. The authors believe that these management changes are directly responsible for the faster resolution of patients' SRC symptoms.

In 2009, SRC guidelines ${ }^{19}$ emphasized that "physical and cognitive rest is required" for recovery while the athlete continues to have symptoms after sustaining an SRC. However, in 2013, SRC guidelines recognized that "the evidence evaluating the effect of rest following an SRC is sparse" and further research to evaluate the optimal and type of rest following an SRC was needed. ${ }^{20}$ Following the 2013 guidelines, ${ }^{20}$ multiple studies and reviews ${ }^{6-10}$ demonstrated that earlier initiation of aerobic exercise was associated with faster full return to sport and school/work. Thus, the latest published guidelines encourage athletes who have sustained an SRC to become gradually and progressively more active within 24 to 48 hours postinjury, while staying below their cognitive and physical symptom-exacerbation thresholds. ${ }^{1,2}$ One of the major differences in SRC management between the athletes in the two data sets is that the 20112013 athletes ${ }^{5}$ were recommended to undergo strict rest per the guidelines of the time, ${ }^{19}$ while the 2016-2018 athletes were advised to engage in active rest. Strict rest is defined as refraining from any physical or cognitive activity until concussion symptoms resolve. Active rest is defined as initiating low intensity physical and cognitive activity at 24 to 48 hours post-SRC, gradually increasing intensity and duration each day provided the activity does not significantly exacerbate symptoms. The activity should have a low risk of head contact/trauma, and active competition should be avoided. Athletes in this study could use their discretion on what he or she could handle mentally and physically. Active rest does not have strict protocols and should not be confused with return to sport (RTS) protocols outlined by SRC consensus statements. ${ }^{1,2}$ RTS protocols should only be started when an athlete has clinically recovered from his or her concussion. This change in 'rest' could have contributed to the significant improvement seen in median SRC symptom duration between the 2011-2013 cohort $^{5}$ (male athletes, 11 days; female athletes, 28 days) and the 2016-2018 cohort (male athletes, 5 days; female athletes, 7 days).

Preexisting conditions, such as migraines and mental health problems, seem to put athletes at greater risk for prolonged symptoms after sustaining a SRC. ${ }^{1,2,11,12}$ Anxiety and depression have specifically been associated with an increased risk for persistent concussion symptoms. ${ }^{2,13,14}$ The presence of convergence insufficiency and vestibular ocular dysfunction in the initial examination following an SRC is a risk factor for prolonged recovery. ${ }^{2,15,16}$ However, vestibular ocular dysfunction findings have been seen in neurologically normal, nonconcussed children. ${ }^{21}$ This raises the question of whether preexisting vision problems could be a risk factor for prolonged SRC symptom recovery. Those with attention deficit hyperactivity disorder or learning disabilities (ADHD/LD) might require more careful planning regarding return to learn but do not appear to be at risk for prolonged symptom recovery. ${ }^{1,2,11}$ However, ADHD/LD has been associated with worse baseline scores on vestibular/ocular motor screening on the baseline, which may carry over into postconcussive vestibular/ocular motor screening scores. ${ }^{17}$

In 2017, we theorized that PPCS could be attributed to the presence of another preinjury medical condition or psychosocial dysfunction. ${ }^{5}$ Preexisting conditions are often dependent on patient self-reporting. If a diagnosis of a preexisting condition has never been given, patients cannot be expected to report the diagnosis. This is especially true in the adolescent age group, as some may have a diagnosis that they are not aware of yet, which makes screening for preexisting conditions more complex in this age group. In the 2011-2013 study, ${ }^{5}$ preexisting conditions were screened for by asking athletes/parents about the presence of exact preinjury diagnoses. The 2016-2018 athletes were screened with a broader approach in asking about preinjury problems. The principal investigator (J.M.N.) has found it much more helpful to ask about past medical history in a different way. For instance, when seeing a patient initially for a concussion, instead of asking "Do you have a history of migraine headaches?," he has found it more useful to ask, "Before this injury happened, how often would you say you got a headache?," If the patient reports regular headaches, follow up questions about the preinjury headaches could uncover the possibility of a preexisting problem (e.g., migraine, vision, anxiety). Other preexisting conditions can be screened for in a similar way. History of preinjury motion sickness from car rides or intolerance to 3D movies could indicate a preexisting vestibular-ocular disorder. ${ }^{22}$ Asking parents about preinjury personality/demeanor may uncover preexisting anxiety. This expansive, individualized, patient-centered, osteopathic approach in screening the athletes' past medical history explains the increase in prevalence in most preexisting conditions between the two studies.

When comparing the $2011-2013^{5}$ and 2016-2018 data sets, there was a higher incidence of preexisting problems for patients in the newer data set, except learning disabilities, suggesting better screening of these conditions. Male athletes showed a statistically significant increase in the incidence of preinjury migraines/frequent headaches and 
mental health problems (anxiety/depression), with a trend toward statistically significant difference in visual problems in the 2016-2018 data sets (Table 4). The 2016-2018 female athletes also showed a statistically-significant increase in the incidence of preinjury migraines/frequent headaches, vision problems, and mental health problems (anxiety/depression; Table 4). In the 2016-2018 study, female athletes, compared with male athletes, showed a statistically significant increase in preinjury incidence of mental health problems (anxiety/depression) and a trending toward statistically significant difference in preinjury incidence of migraine/frequent headaches and vision problems (Table 1). When preexisting conditions were identified on initial examination, symptom recovery tended toward be prolonged regardless of sex, except for a significant difference associated with isolated mental health conditions favoring girls (Table 2). This data set suggests that previous publication findings of longer recovery of concussion symptoms in female athletes may be related to a higher incidence of preexisting conditions, diagnosed or not, when compared with male athletes.

Identifying preexisting conditions on initial evaluation can help improve SRC recovery. SRC could potentially exacerbate a preexisting condition. Identifying a potential preexisting condition may influence a practitioner's decision to institute a treatment sooner during SRC management. For instance, the threshold for sending an athlete to vestibular therapy after sustaining an SRC could be lower in an athlete who describes a history of frequent preinjury motion sickness when compared with athletes without that preinjury history. Additionally, identifying preexisting conditions on initial evaluation could better establish the presence of such symptoms before the concussion, which prevents them from being mistakenly attributed as ongoing concussion symptoms at follow-up visits.

The most consistent predictor of slower recovery from concussion is the severity of a person's acute/subacute symptoms. ${ }^{1,2,11}$ Increased preinjury somatization of symptoms at baseline among athletes has demonstrated an association with higher acute symptoms scores and protracted recovery if and when they sustain a concussion. ${ }^{3}$ Other publications have suggested that catastrophizing the injury may increase anxiety and affect concussion recovery. ${ }^{4}$ Earlier initiation of clinical care is associated with faster recovery after concussion. ${ }^{18}$ Early patient counseling and education about the realities of concussion and the normal course of symptom resolution may mitigate anxiety and improve concussion recovery. This counseling is probably even more critical to patients with preexisting anxiety. Practitioners should remain optimistic, as the vast majority of concussions resolve without long-term sequela.
In the 2011-2013 data set, ${ }^{5}$ concussion knowledge was significantly less, and physician counseling often had a much more cautious, or at times ominous, tone. An optimistic outlook is important for concussion patients and making clinic visits more positive can contribute to quicker concussion recovery.

\section{Limitations}

Due to the nature of a retrospective medical record review, the timeline for scheduling initial postinjury concussion evaluations and follow-up appointments could not be standardized. The appointment scheduling variable was not constant and may have altered the actual length of recovery. Another limitation of this study lies within the diagnosis of concussion. Physicians must rely on patients to self-report their symptoms, contributing a subjective nature to the condition. This subjectivity can interfere with the monitoring and assessing a patient's concussion, thereby altering the data set. This limitation is a concern with most concussion research.

A specific limitation of this study was that preexisting conditions were not stringently quantified. Preinjury history was more focused on generally assessing for preexisting problems rather than specific diagnoses. The frequency and severity of preexisting problems were not documented. The presence of a preexisting problem suggests the possibility of a preexisting medical condition, but is not conclusive in the absence of a formal diagnosis before his or her injury. Finally, the new data set from athletes seen from 2016-2018 includes patients seen by a single practitioner (J.M.N.), whereas the data set from $2011-2013^{5}$ included patients seen by three different practitioners (including J.M.N.).

\section{Conclusion}

This study's findings show that initiating of "active rest" following SRC, recognizing the impact of preexisting conditions, and educating patients about recovery expectations can decrease symptom duration in first-time SRC in middle school and high school athletes. Further research, including prospective studies, is needed to establish which of these measures is most critical in decreasing symptom duration. The discovery of concussion biomarkers may better understand the mechanism of injury of concussion and the physiology of its associated subjective symptoms.

Acknowledgments: The authors thank Godwin Dogbey, $\mathrm{PhD}$, of Campbell University School of Osteopathic Medicine for statistical analysis. 
Research funding: None declared.

Author contributions: All authors provided substantial contributions to conception and design, acquisition of data, or analysis and interpretation of data; all authors drafted the article or revised it critically for important intellectual content; all authors gave final approval of the version of the article to be published; and all authors agree to be accountable for all aspects of the work in ensuring that questions related to the accuracy or integrity of any part of the work are appropriately investigated and resolved.

Competing interests: Authors state no conflict of interest. Informed consent: Informed consent was unnecessary for this retrospective chart analysis.

Ethical approval: The Campbell University Institutional Review Board deemed this study exempt.

\section{References}

1. McCory P, Meeuwisse WH, Dvorak J, et al. Consensus statement on concussion in sport: the 5th international conference on concussion in sport held in Berlin, October 2016. Br J Sports Med. 2017;51(11):838-847.

2. Harmon KG, Clugston JR, De K, et al. American Medical Society for Sports Medicine position statement on concussion in sport. $\mathrm{Br}$ J Sports Med. 2019;53:213-225.

3. Nelson LD, Tarima S, LaRoche AA, et al. Preinjury somatization symptoms contribute to clinical recovery after sports-related concussion. Neurology. 2016;86(20):1856-63.

4. Greenberg J, Mace RA, Funes CJ, et al. Pain catastrophizing and limiting behavior mediate the association between anxiety and postconcussion symptoms. Psychosomatics. 2019;(19)30192-6.

5. Neidecker JM, Gealt DB, Luksch JR, Weaver MD. First-time sportsrelated concussion recovery: The role of sex, age and sport. J Amer Osteo Assoc. 2017;117(10):635-642.

6. Lawrence DW, Richards D, Comper P, et al. Earlier time to aerobic exercise is associated with faster recovery following acute sport concussion. PLoS One. 2018; (4)e0196062.

7. Leddy JJ, Haider MN, Ellis MJ, et al. Early subthreshold aerobic exercise for sport-related concussion: a randomized clinical trial. JAMA Pediatr. 2019;(4):319-325.

8. Thomas DG, Apps JN, Hoffman RG, et al. Benefits of strict rest after acute concussion: a randomized control trial. Pediatrics. 2015;(2): 213-23.
9. Makdissi M, Schneider KJ, Feddermann-Dermont N, et al. Approach to investigation and treatment of persistent symptoms following sport-related concussion: a systematic review. $\mathrm{Br} J$ Sports Med. 2017;(12):958-968.

10. Willer BS, Haider MN, Bezherano I, et al. Comparison of rest to aerobic exercise and placebo-like treatment of acute sportrelated concussion in male and female adolescents. Arch Phys Med Rehabil. 2019;(12):2267-2275.

11. Iverson GL, Gardner AJ, Terry DP, et al. Predictors of clinical recovery from concussion: a systematic review. Br J Sports Med. 2017;(12):941-948.

12. Zemek R, Barrowman N, Freedman SB, et al. Clinical risk score for persistent postconcussion symptoms among children with acute concussion in the ED. JAMA. 2016;(10):1014-1025.

13. Dwyer B, Katz D. Postconcussion syndrome. Handb Clin Neurol. 2018;(158):163-178.

14. Ponsford J, Nguyen S, Downing M, et al. Factors associated with persistent post-concussion symptoms following mild traumatic brain injury in adults. J Rehabil Med. 2019;(1): 32-39.

15. Ellis MJ, Cordingley DM, Vis S, et al. Clinical predictors of vestibulo-ocular dysfunction in pediatric sports-related concussion. J Neurosurg Pediatr. 2017;(1):38-45.

16. DuPrey KM, Webner D, Lyons A, et al. Convergence insufficiency identifies athletes at risk of prolonged recovery from sport-related concussion. Am J Sports Med. 2017;(10): 2388-2393.

17. Moran RN, Wallace J, Murray NG, Covassin T. Effects of attention deficit hyperactivity and learning disability on vestibular and ocular baseline concussion assessment in pediatric athletes. Appl Neuropsychol Child. 2019;1-7.

18. Kontos AP, Jorgensen-Wagers K, Trbovich AM, et al. Association of time since injury to the first clinic visit with recovery following concussion. JAMA Neurol. 2020 Jan 6.

19. McCrory P, Meeuwisse W, Johnston K, et al. Consensus statement on concussion in sport - the third international conference on concussion in sport held in Zurich, November 2008. Phys Sportsmed. 2009;(37):141-159.

20. McCrory P, Meeuwisse W, Aubry M, et al. Consensus statement on concussion in sport - the 4th international conference on concussion in sport held in Zurich, November 2012. Br J Sports Med. 2013;(47):250-258.

21. Corwin DJ, Zonfrillo MR, Wiebe DJ, et al. Vestibular and oculomotor findings in neurologically-normal, non-concussed children. Brain Inj. 2018;(6):794-799.

22. Solimini AG. Are there side effects to watching 3D movies? A prospective observational study on visually induced motion sickness. PLoS One. 2013;(2) e56160. 